\title{
Study of the exploration focused on shear waves generated by a dipole source
}

\author{
Yuki NAGAI ${ }^{1}$, Akinori TSUDA ${ }^{1}$, Hiroaki HATANAKA ${ }^{1}$, Minoru TAGAMI ${ }^{1}$, \\ Koichiro TANAKA ${ }^{1}$, Fumio SATO $^{1}$, Junichi TAKEKAWA ${ }^{2}$ and Hitoshi MIKADA ${ }^{2}$ \\ ${ }^{1}$ IHI Corporation \\ ${ }^{2}$ Kyoto University
}

\begin{abstract}
In this paper, we focused on the generation of the shear wave using a dipole source in water. We made a scaled model of a deep-tow seismic survey using solid material with a set of reflectors and an overlain water layer. Two dilatational sources with the opposite phase to each other are simultaneously excited to form a point force in the water parallel to solid-water interface. The sound field generated by the dipole in water creates a stress field tangential to the solid-water interface so that the shear wave is generated in the solid. For the model, we performed both acoustic experiment and numerical simulations to see both compressional and shear reflection profiles. Numerical simulation indicates that the amplitudes of the S-to-S or S-to-P reflected would be emphasized if we use horizontal seismic sensors on the fluid-solid interface. Although the signal-to-noise ratio of the acoustic experiment is not sufficiently high, we confirmed that shear reflection profile could be produced. It should be noted that shear waves could be produced in water using multipolar sources fired close to the seafloor, and that some improvements to increase shear wave energy more than the compressional wave need to be pursued for practical use of multipolar sources.
\end{abstract}

\section{INTRODUCTION}

For the exploration and the development of the hydrocarbon fluid resources such as oil, natural gas, etc., a 3D seismic exploration method has been applied to investigate subsurface structures. In particular, the utilization of airguns and multiple streamers have made use of 3D surveys in practice in the marine environment. Marine seismic sources are in general explosive or impulsive so that the compressional and its mode converted waves have been used in the exploration. For further effective exploitation of the resources, such as enhanced oil recovery, it is necessary to apply much higher exploration technologies. Shear waves are also a candidate to be utilized to investigate physical properties of subsurface materials. Therefore, shear waves have been of interest in research in recent years ${ }^{1)}$, but, unfortunately, are known as difficult to be acquired in the marine environment due to water layer in which no shear wave travels through.

For the purpose of the reduction of impact to marine mammals, the industry has initiated a joint industrial project for the realization of marine vibratory seismic source ${ }^{2-4)}$. Because of controllable nature of such seismic sources, it would become possible to produce seismic signals with repeatability. Although repeatable seismic signals could surely enhance the signal-to-noise ratio in the analysis of time-lapse seismic surveys frequently applied in the marine environment, it has not been well considered to produce shear waves using marine vibratory sources, nor the generation of shear waves without touching the seafloor. For efficient surveys using shear waves, we need to find an alternative way to generate shear waves and to exploit the controllable nature of marine vibratory sources.

In this paper, the results of a study of the exploration focused on the generation of shear waves using a dipole source above the seafloor are shown. We conducted both marine environment and scaled experiments to confirm that shear waves could be generated using towed seismic sources and that reflection profiles could be produced.

\section{THE PRINCIPLE OF THE DIPOLE SOUND SOURCE}

Two dilatational sources with the opposite phase to each other are simultaneously excited to form a point force in the water parallel to solid-water interface. The sound field generated by the dipole in water creates a stress field tangential to the solid-water interface so that the shear wave is 
generated in the solid. Figure 1 shows the schematic image of the shear wave exploration by the dipole sound source.

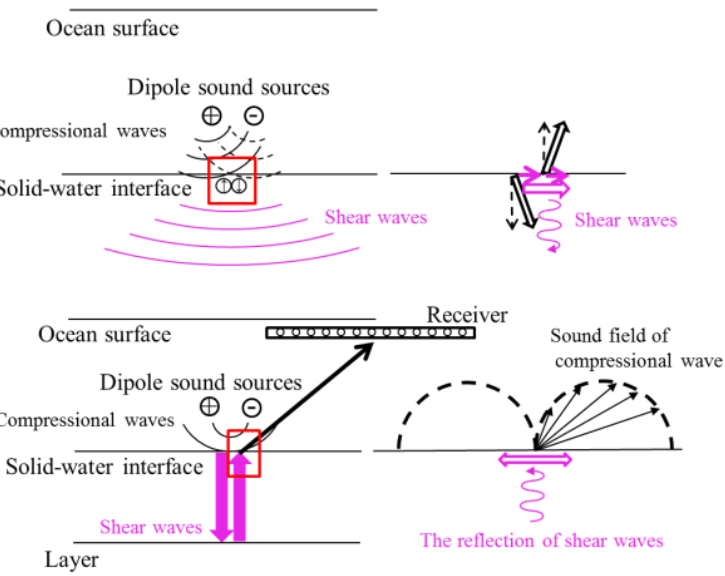

Figure 1 Schematic image of exploration using the dipole sound source

\section{NUMERICAL SIMULATION}

In this examination, we performed the numerical simulation for the shear wave exploration by using the dipole source. Figure 2 shows the simulation model in the marine environment. Mortar was introduced to emulate the underground in oceans and the sand layers which emulated oil or gas layers were installed in it. As for the sound source, $50 \mathrm{~Hz}$ frequency was employed, and the sources were set $60 \mathrm{~m}$ above the mortar surface, and two sound sources with the opposite phase to each other were simultaneously excited. The receivers were contacted the mortar surface, and acquired the horizontal displacement and acoustic pressure. The distances between receivers were set aligned with $12.5 \mathrm{~m}$ spacing. For the simulation, acoustic velocities of compressional and shear waves, density, and attenuation rate were measured as shown in Table 1. Figure 3 and Figure 4 show the result of the simulation. Figure 3 shows the surface displacement of horizontal motion, and Figure 4 shows the acoustic pressure received the mortar surface. As the result of the simulation, the reflection of the compressional wave from the upper sand layer(I) was able to be detected, but the reflections from the lower sand layer(III) could not be recognized. On the other hand, the reflection of the shear wave was higher than that of the compressional wave from the upper sand layer(I), and the reflections from the lower sand layer(III) were able to be detected. Because the shear wave did not appear in the acoustic pressure, the signal intensity of the waves from the sand layers was weak as shown Figure 4.

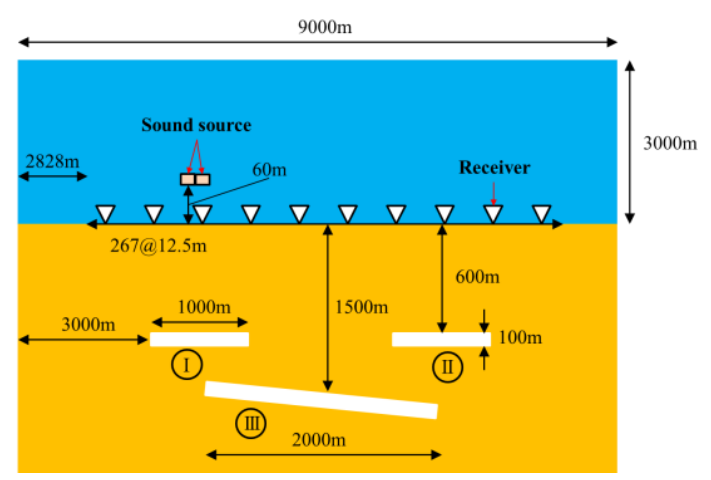

Figure 2 The simulation model

Table 1 The simulation condition

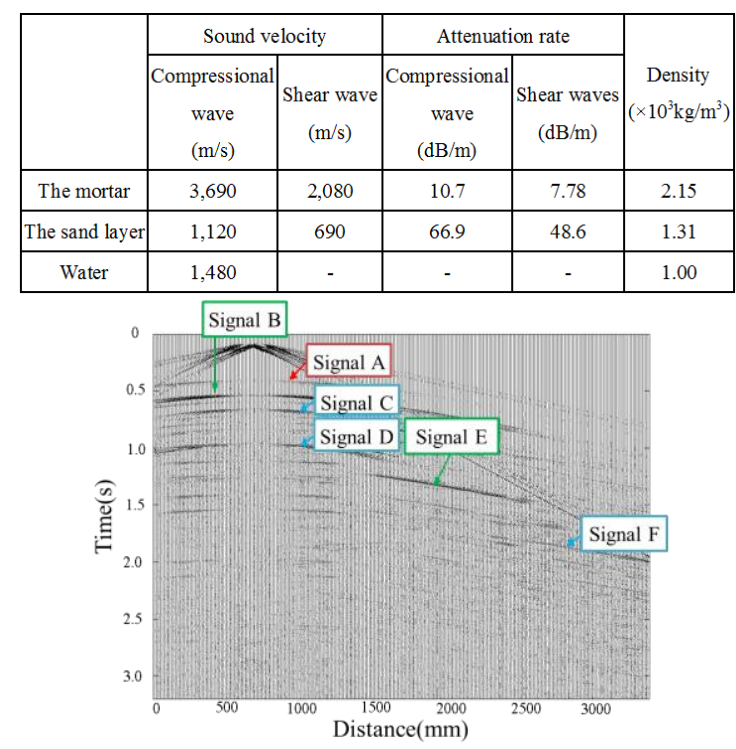

Figure 3 The horizontal displacement, Signal A; The compressional wave from the top of the sand layer(I), Signal B; The mode conversion wave from the top of the sand layer(I), Signal $C$; The shear wave from the top of the sand layer(I), Signal D; The shear wave from the bottom of the sand layer(I), Signal E; The mode conversion wave from the top of the sand layer(III), Signal F; The shear wave from the top of the sand layer(III)

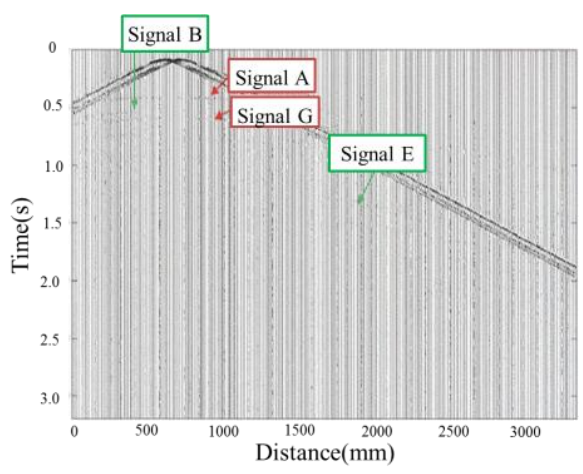

Figure 4 The acoustic pressure, Signal G; The compressional wave from the bottom of the sand layer(I) 
In the next place, we performed the numerical simulation for the shear wave exploration in the scaled model as shown in Figure 5. As for the sound source, 1 wavelength with $100 \mathrm{kHz}$ frequency were employed, and the sound sources were set $30 \mathrm{~mm}$ above the mortar surface ( 2 wavelengths in water: $29.6 \mathrm{~mm}$ ). The distances between two sound sources and a receiver were set aligned with $50 \mathrm{~mm}$ spacing, and two sound sources of the opposite phase were simultaneously excited. The interface $\mathrm{A}$ is the interface between water and the mortar, the B, C, D, and $\mathrm{E}$ are the interface between the mortar and the sand layers. We calculated the wave propagation by using the finite element method. Figure 6 shows the signal intensity of the reflection waves from each interface, respectively. As the result of the simulation, the reflection of the compressional wave from the lower sand layer (D) could not be recognized, but the reflections of the shear waves from the lower sand layer (D and E, as well) were able to be detected. We confirmed that the shear wave propagates with lower attenuation than the compressional wave in the mortar and the sand layers.

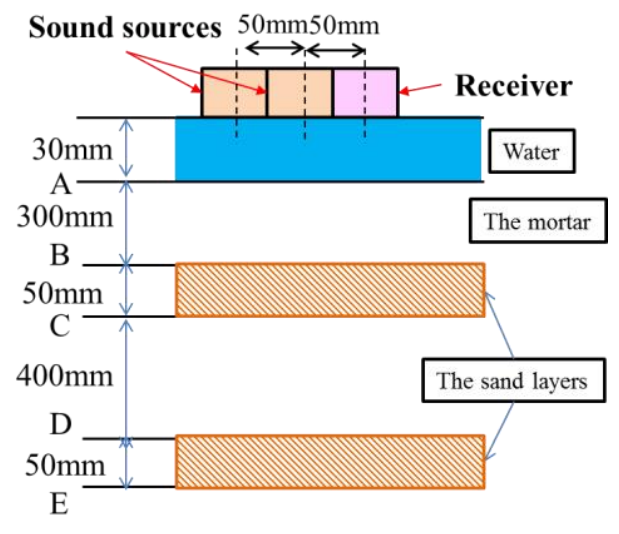

Figure 5 The result of simulation (Reflection wave)

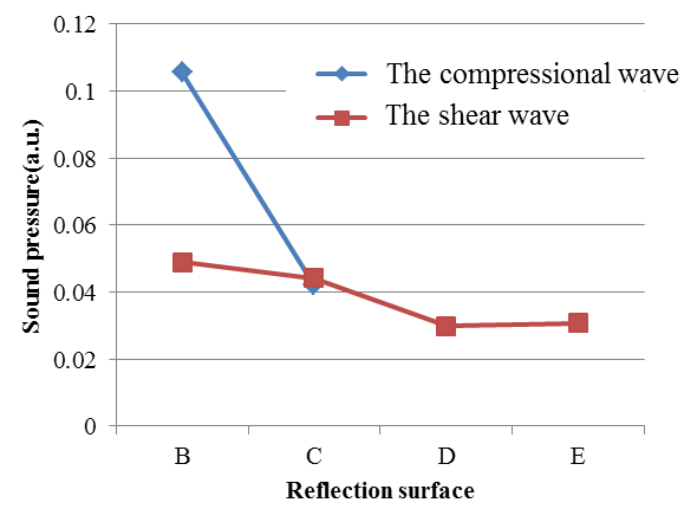

Figure 6 The result of simulation (The signal intensity of the reflection waves)

\section{EXPERIMENT}

The specimen of the ocean area reduction model was prepared to conduct the fundamental test for the exploration of oilfields by the shear waves. The dimension of mortar specimen is $2000 \mathrm{~mm}^{\mathrm{w}} \times 2000 \mathrm{~mm}^{1} \times 1500 \mathrm{~mm}^{\mathrm{h}}$ and three sand layers were installed in the specimen as shown Figure 7. The sound source which has a frequency of $100 \mathrm{kHz}$ and two sources were set $30 \mathrm{~mm}$ above the mortar surface. To emulate the exploration using streamer cables in oceans, the sound sources and the receiver were scanned with certain distances. The distances were set between $50 \mathrm{~mm}$ and $450 \mathrm{~mm}$ with a $6.25 \mathrm{~mm}$ increment as shown in Figure 8 (assuming the exploration frequency was $50 \mathrm{~Hz}$ in the marine environment, the distances were $12.5 \mathrm{~m}$ corresponding to wavelength ratio). The guide rail was established on the mortar specimen with two sound sources and one receiver to scan them.

(a)
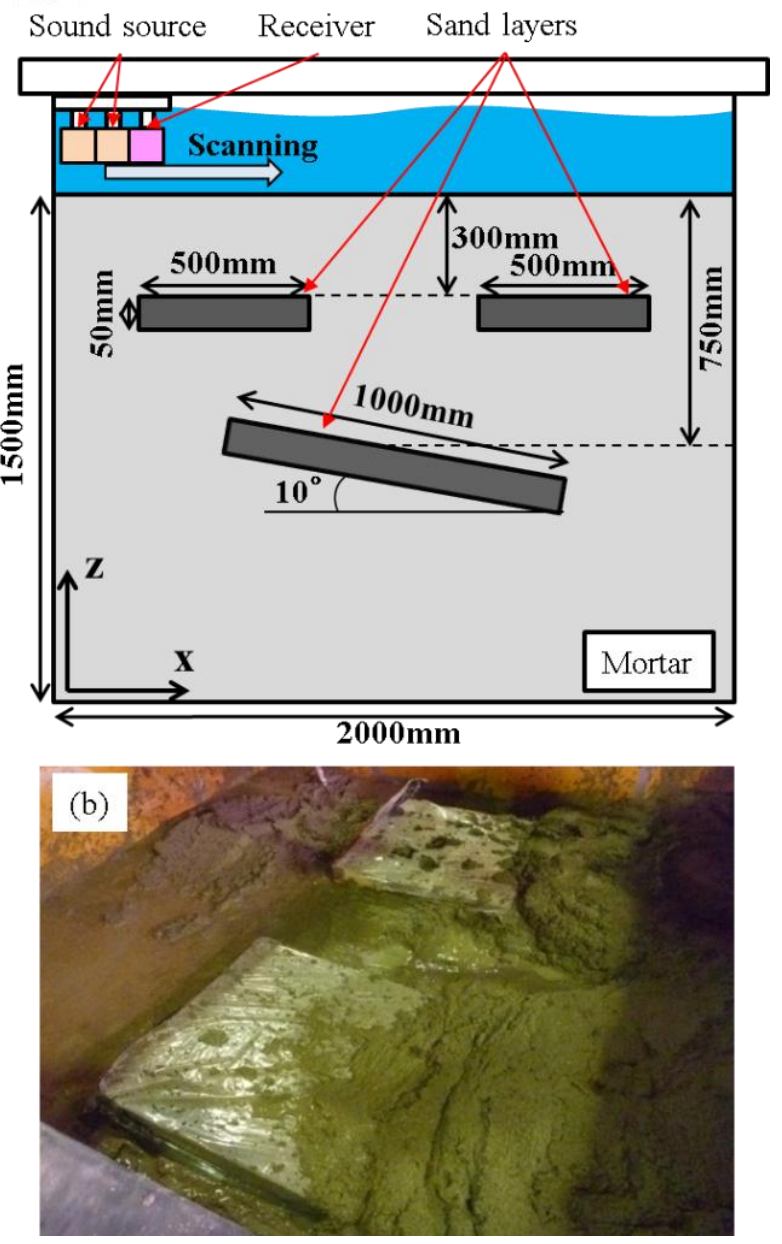

Figure 7 (a) The specimen layout and (b) being installed sand layers 

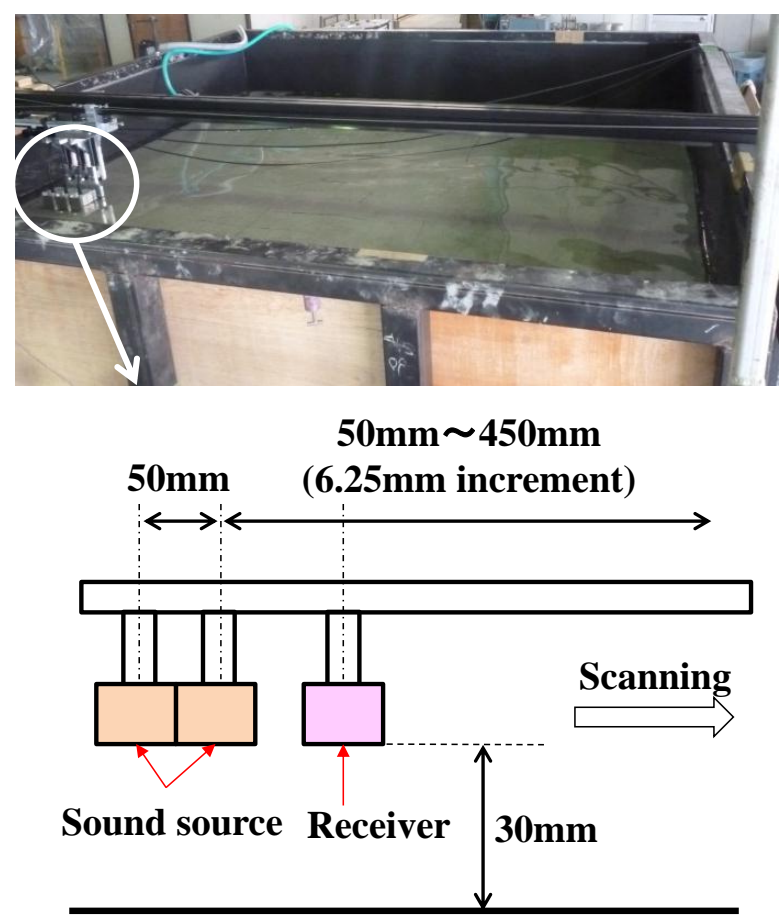

The specimen surface

Figure 8 The guide rail, dipole sound source and a receiver

Position(mm)

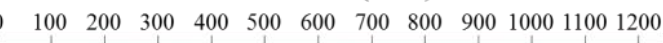

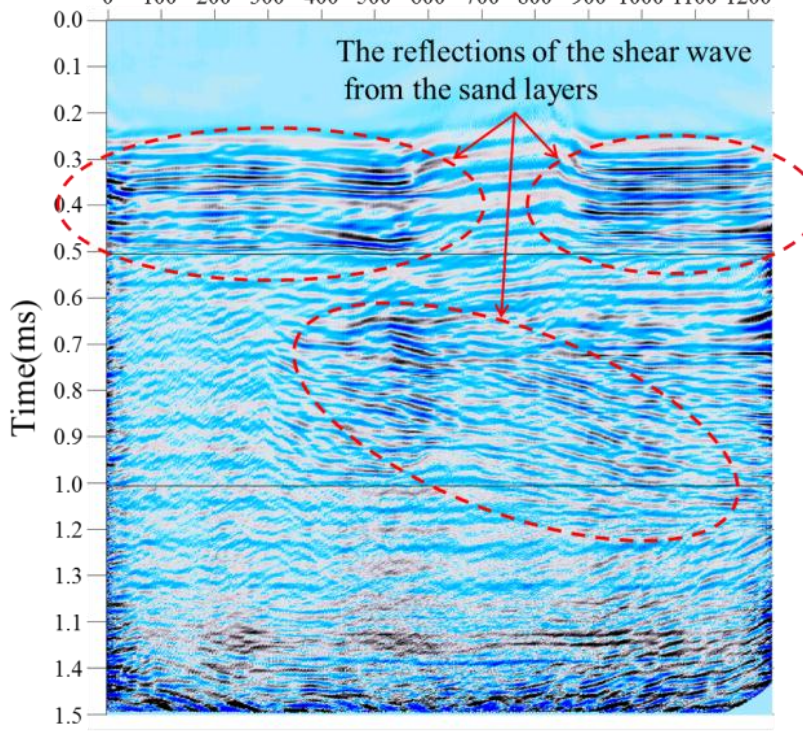

Figure 9 The result of the exploration with the mortar specimen

After the data acquisition, we employed ActiveSeisDPS software made by JAPEX Geoscience Institute, Inc. for the signal processing in this examination. We compensated the amplitude by $30 \mathrm{~dB} / \mathrm{ms}$. first, and the deconvolution was applied to make broad reflections sharp. To remove reflections from the side wall of the specimen, Radon conversion was introduced. Subsequently, the velocity analysis, normal move out correction and common midpoint stacking were conducted.

Figure 9 shows the result of the exploration for the mortar specimen by using the dipole sound source and signal processing focused on shear waves. As a result, the reflections of the shear wave from the bottom of the sand layers were able to be detected, although the bottom of the sand layers was not able to detect by compressional waves.

\section{CONCLUSION}

A shear wave exploration was attempted using both a marine environment and a scaled model. The marine environment numerical model was used to simulate a multi-component sensor system permanently installed on the seafloor, while the scaled model consists of mortar and buried three sand layers. The sand layers emulate oil or gas layers that were used as reflectors to see if they are detectable by a dipole sound source. The S-to-S reflections were observed both in marine environment numerical model and in the scaled experiment. We examined the signal-to-noise ratio for the two experiments and found out that S-to-S reflections would be predominant signals in the record of the horizontal component of the seafloor-installed sensors. Our results are pretty encouraging in a sense that towed seismic sources in water could produce shear waves beneath the seafloor when they are used as a multipole source fired above the seafloor. We would like to continue our researches to pursue a practical use of multipolar sources.

\section{REFERENCES}

1) Barkved, O., Bartman, B., Compani, B., Gaiser, J., Dok, R.V., Johns, T., Kristiansen, P., Probert, T., Thompson, M., 2004, The Many Facets of Multicomponent Seismic Data, 16 (2), Oilfield Review, 42-56.

2) Tenghamn, R., 2006, PGS shows off electrical marine vibrator to capture 'alternative' seismic source market, First Break, 24 (1), 33-36.

3) Goertz, A., J.F. Wisløff, F. Drossaert and J. Ali, 2013, Environmental source modelling to mitigate impact on marine life, First Break, 31 (11), 59-64.

4) Pramik, B., Marine Vibroseis: shaking up the industry, First Break, 31 (11), 67-72. 